\title{
Th2-like immune response in radiation-induced lung fibrosis
}

\author{
GUANG HAN $^{1-3^{*}}$, HUI ZHANG ${ }^{1,2^{*}}$, CONG HUA XIE ${ }^{1,2^{* *}}$ and YUN FENG ZHOU ${ }^{1,2^{* *}}$ \\ ${ }^{1}$ Department of Radiation and Medical Oncology, and ${ }^{2}$ Hubei Key Laboratory of Tumor Biological Behaviors, \\ Zhongnan Hospital, Wuhan University, Wuhan $430071 ;{ }^{3}$ Department of Radiotherapy, \\ Hubei Cancer Hospital, Wuhan, P.R. China
}

Received January 19, 2011; Accepted March 10, 2011

DOI: $10.3892 /$ or.2011.1300

\begin{abstract}
Pulmonary fibrosis is a common delayed side effect of radiation therapy. Since its mechanism is almost unknown, little can be done to prevent or treat it. Th2 cytokines have been clearly implicated as mediators of asthma, and evidence is mounting that type 2 immune responses may also promote the development of pulmonary fibrosis. The aim of this study was to investigate whether Th2-like immune responses account for the development and progression of chronic radiation pulmonary fibrosis. C57BL/6 mice received thoracic irradiation of $12 \mathrm{~Gy}$ and were sacrificed at $1 \mathrm{~h}$ and 1, 2, 4, 8, 16 and 24 weeks post-irradiation (p.i.). We assayed the expression of IL-13 in serum, and the expression of hydroxyproline and the mRNA and protein of GATA-3 and Arg-1 in lung tissue. mRNA and protein analysis revealed the expression of these Th2-immune response-associated factors (GATA-3, IL-13 and Arg-1) in mice after irradiation. Without causing conspicuous fibrotic pathological changes at the early post-irradiation phase ( 1 and 2 weeks p.i.), a Th2 profile was confirmed by significantly elevated expression of Th2-specific transcription factor GATA-3 mRNA $(\mathrm{P}<0.01)$. Protein analysis confirmed the GATA-3 mRNA expression. Following significantly elevated expression of hydroxyproline $(\mathrm{P}<0.01)$ at 16 weeks p.i., IL-13 and Arg-1 expression reached maximal values in serum and lung tissue and maintained high levels up to 24
\end{abstract}

Correspondence to: Dr Cong Hua Xie, Department of Radiation and Medical Oncology and Hubei Key Laboratory of Tumor Biological Behaviors, Zhongnan Hospital, Wuhan University, 169 Dong Hu Road, Wuhan, Hubei 430071, P.R. China

E-mail: xch1965@hotmail.com

*Contributed equally

** Joint senior authorship

Abbreviations: p.i., post-irradiation; RILF, radiation-induced lung fibrosis; Th, T helper; GATA-3, GATA-binding protein 3; Arg-1, arginase 1; SPF, specific pathogen-free; SSD, source-surface distance; RT-PCR, real-time quantitative reverse transcriptase PCR

Key words: radiation-induced lung fibrosis, Th2-like immune response, GATA-binding protein, interleukin-13, arginase-1 weeks p.i., respectively $(\mathrm{P}<0.01)$. Our data indicate that lung irradiation induces Th2 polarization. Furthermore, Th2-like immune response may take part in radiation-induced pulmonary fibrosis (RILF), and GATA-3 may play an important role in promoting RILF. Thus, GATA-3 may be an important target for the treatment of RILF.

\section{Introduction}

Radiation-induced lung fibrosis (RILF) is a common delayed side effect of radiotherapy for thoracic malignancies, which limits the delivered dose to the tumor target and may thus hamper tumor control (1). RILF is believed to result from cytokine-mediated multicellular interactions with induction and progression of fibrotic tissue reactions $(2,3)$; however, the mechanism(s) underlying the pathogenesis of RILF at the molecular and cellular levels has not yet been identified.

Naive $\mathrm{CD} 4^{+} \mathrm{T}$-helper (Th) cells can be differentiated into at least two functional subsets during the immune response: Th1 cells, which secrete Th1 cytokines such as INF- $\gamma$, TNF- $\beta$, IL-2, IL-12; and Th2 cells which secrete Th2 cytokines, such as IL-4, IL-5, IL-6, IL-10 and IL-13 (4). Th1 and Th2 cytokines are crucial to Th1 and Th2 immune reactions, which both promote the growth/differentiation of their subset and inhibit the growth/differentiation of the opposing subset (5). Th2-dominated immune responses secrete multiple Th2 cytokines, key players in humoral immunity. They also play a central role in the pathogenesis of a variety of fibrotic disorders, including progressive systemic sclerosis, idiopathic pulmonary fibrosis, bleomycin lung and hepatic fibrosis (6-8). Recent studies have shown that several Th2 cytokines are expressed in RILF. Buttner et al (9) and Huaux et al (10) reported elevated levels of the type 2 cytokine interleukin (IL)- 4 in rats during RILF. Tabata et al (11) showed that all-trans-retinoic acid prevents RILF by suppressing the expression of IL-6 in mice lung tissue after irradiation. IL-10 was also found to be released in RILF (12). Yang found that increased expression of IL-13 and IL-4 are associated with an earlier onset and enhanced degree of RILF (13). Although these studies provide little more than vignettes of the molecular events in the lung after irradiation, they provide some useful hypotheses as to the role of Th2-like immune response in RILF.

Collagen, elastin and a component of complement are proteins that contain hydroxyproline. Since collagen is by far 
the most abundant protein in the lung, comprising $60-70 \%$ of the tissue mass, analysis of the hydroxyproline content in lung tissues provides a reliable quantitative index for pulmonary fibrosis (14).

GATA-binding protein 3 (GATA-3) was first identified as a T-cell-specific transcription factor in 1991 by Ko and Engel (15). GATA-3 plays a pivotal role in the development of the Th2 phenotype while inhibiting Th1 cells and producing type 2 cytokines (16). IL-13, a Th2 cell hallmark cytokine, is regarded as a strong inducer of tissue fibrosis $(17,18)$. Studies have shown that IL-13 is regulated by GATA-3 and the IL-13 promoter is mediated by a functionally critical GATA-3 site that binds endogenous GATA-3 proteins, resulting in an increase in IL-13 production $(19,20)$. Arginase 1 (Arg-1), an enzyme that catalyzes the hydrolysis of arginine during the urea cycle, produces urea and L-ornithine. L-ornithine is further metabolized by ornithine decarboxylase and ornithine amino transferase, and produces polyamines and L-proline, which control cell growth and collagen synthesis, respectively. Thus, Arg-1 is required for tissue remodeling and fibrosis $(21,22)$. Studies have shown that Th2-cytokines up-regulate Arg-1 expression in vivo and in vitro $(23,24)$. From these observations, it appears that GATA-3 plays a pivotal role in the Th2-like immune response, and produces type 2 cytokines to regulate Arg-1 expression and hydroxyproline content.

In order to investigate the relationship between the Th2-like immune response and pathogenesis of RILF, C57BL/6 mice received thoracic irradiation, and the levels of associated factors (GATA-3, IL-13 and Arg-1) and hydroxyproline content were measured in lung tissue and serum at various times from $1 \mathrm{~h}$ to 24 weeks after irradiation.

\section{Materials and methods}

Animals and radiation schedule. Specific pathogen-free (SPF) C57BL/6 mice were purchased from Vital River Laboratories (Beijing, China). Eight-week-old female mice ( $20 \mathrm{~g}$ each) were divided into 2 groups: i) non-irradiated control group (14 mice) and ii) radiation group (35 mice). A dose of 12 Gy to the mid-plane of the lungs was administered in a single fraction via a posterior field using a linear accelerator (Siemens Primus-Hi). A plastic jig was used to restrain the mice without anesthesia, and lead blocks were placed to shield the head and abdomen. Characteristics of the radiation were as follows: beam energy, 6-MV photons; dose rate, $1.886 \mathrm{~Gy} / \mathrm{min}$; source-surface distance (SSD), $1 \mathrm{~m}$; size of the radiation field, $2.6 \times 38 \mathrm{~cm}$. The depth of the maximum dose of the 6-MV photon beam was significantly reduced by the tissue-equivalent plastic material (thickness $10 \mathrm{~mm}$ ) of the restraining jig. Film dosimetry was used to determine the relative dose distribution. Dosimetry was performed with a cylindrical ionization chamber. Following irradiation, mice in the two groups were maintained in an SPF environment and provided with a standard diet and water. The experimental protocols were approved by the Medical Sciences Animal Care Committee of Hubei Province, China.

Tissue isolation. Radiated and control mice were humanely sacrificed at $1 \mathrm{~h}$ and $1,2,4,8,16$ and 24 weeks post-irradiation (p.i.). The left lung lobes were used for histological, histochemical and hydroxyproline content analyses, and the right lobes were quickly frozen in liquid nitrogen until RNA and protein isolation. Sera samples were used for ELISA.

Histology. Paraffin-embedded tissues were sectioned at an average thickness of $4 \mu \mathrm{m}$. Tissue staining was carried out using hematoxylin and eosin (H\&E) to determine histological changes, and Masson's stain was used for collagen detection.

Immunohistochemistry. Tissue dewaxing in xylene and rehydration in graded alcohol were performed according to the manufacturer's protocol. The specimens were incubated overnight at $4^{\circ} \mathrm{C}$ with 100 -fold-diluted rabbit polyantibody against Arg-1 (Santa Cruz Biotechnology Inc., Santa Cruz, CA, USA) and goat anti-rabbit secondary antibody (Maixin Biotechnology, Fuzhou, China).

Hydroxyproline determination. Lung tissue samples were stored at $-80^{\circ} \mathrm{C}$ until analysis. To measure the content of hydroxyproline in the lung tissue, alkaline hydrolysis assay kits (25) (Jiancheng Biological Institution, Nanjing, China) were used according to the instructions provided by the manufacturer. The quantity of hydroxyproline was determined based on the following formula: Content of hydroxyproline $(\mu \mathrm{g} / \mathrm{mg}$, wet weight $)=\left[\right.$ absorbance $_{(\text {sample })}-$ absorbance $\left._{(\text {blank }}\right] /$ [absorbance $_{\text {(standard) }}-$ absorbance $\left._{\text {(blank) }}\right]$ x $5 \mu \mathrm{g} / \mathrm{ml} \mathrm{x} 10 \mathrm{ml} /$ wet weight $_{\text {(tissue) }}$.

ELISA assay. IL-13 concentrations were measured in mouse serum using ELISA kits (eBioscience, San Diego, CA, USA) following the manufacturer's protocols. Purified murine IL-13 from the ELISA kits was used to generate the standard curves for calculation of the cytokine concentrations in each serum sample. Microtiter plates (96-well) were read at $450 \mathrm{~nm}$ in an automated microplate reader. The detection limits of these assays were $4 \mathrm{pg} / \mathrm{ml}$ for IL-13.

RT-PCR analysis. Total RNA was extracted using the TRIzol reagent according to the manufacturer's protocol (Invitrogen, Carlsbad, CA, USA). RNA integrity was assessed using denaturing agarose gel electrophoresis. First-strand cDNA

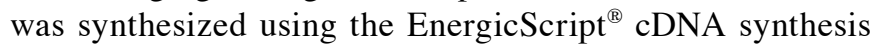
kit (ShineGene, Co., Ltd., Shanghai, China) according to the manufacturer's protocol. RT-PCR was performed as described in a previous report (26). PCR primers and probes for murine GATA-3, Arg-1 and the housekeeping genes, such as GAPDH, were designed by ShineGene Co., Ltd., based on cDNA sequences obtained from the GenBank database (Table I). RT-PCR amplifications were performed using the ShineProbe ${ }^{\circledR}$ Real-Time qPCR MasterMix kits (ShineGene) in the FTC-2000 sequence detection system (Funglyn Biotech Inc., Toronto, Canada). For reproducibility within and between PCR amplifications, we used a standard normal cDNA in each PCR amplification. Relative mRNA expression of test cDNA samples was referenced to the standard cDNA and expressed as the ratio of the level of the test mRNA compared to the base mRNA. For example, relevant mRNA expression $(\mathrm{E})=$ $2^{-(\mathrm{Ct} \text { of test }-\mathrm{Ct} \text { of GAPDH) }}$, where $\mathrm{Ct}$ represents the cycle threshold in the PCR. 
Table I. Primer and probe sequences for murine GATA-3, Arg-1 and GAPDH.

Name

Sequence $\left(5^{\prime}-3^{\prime}\right)$

Amplicon length (bp)

\begin{tabular}{ll}
\hline GATA-3 & FW: ACTGCGGGGCAACCTCTA \\
& RV: CGGTTCTGCCCATTCATTTT \\
& FP: CCACTGTGGCGGCGAGATGGTAC \\
& FW: TTGATTCCAACGACATCTACCA \\
RV: CGTTTCCATTAGCTCCTTCAT & FP: CTGCCTACCGCCTGGGCTCTGA \\
& FW: TGTGTCCGTCGTGGATCTGA \\
GAPDH & RV: CCTGCTTCACCACCTTCTTGA \\
& FP: CCGCCTGGAGAAACCTGCCAAGTATG
\end{tabular}

Forward (FW) and reverse (RV) primers were always located in different exons. Fluorogenic probes (FP) were FAM-labelled at the 5'-end and TAMRA-labelled at the 3 '-end.

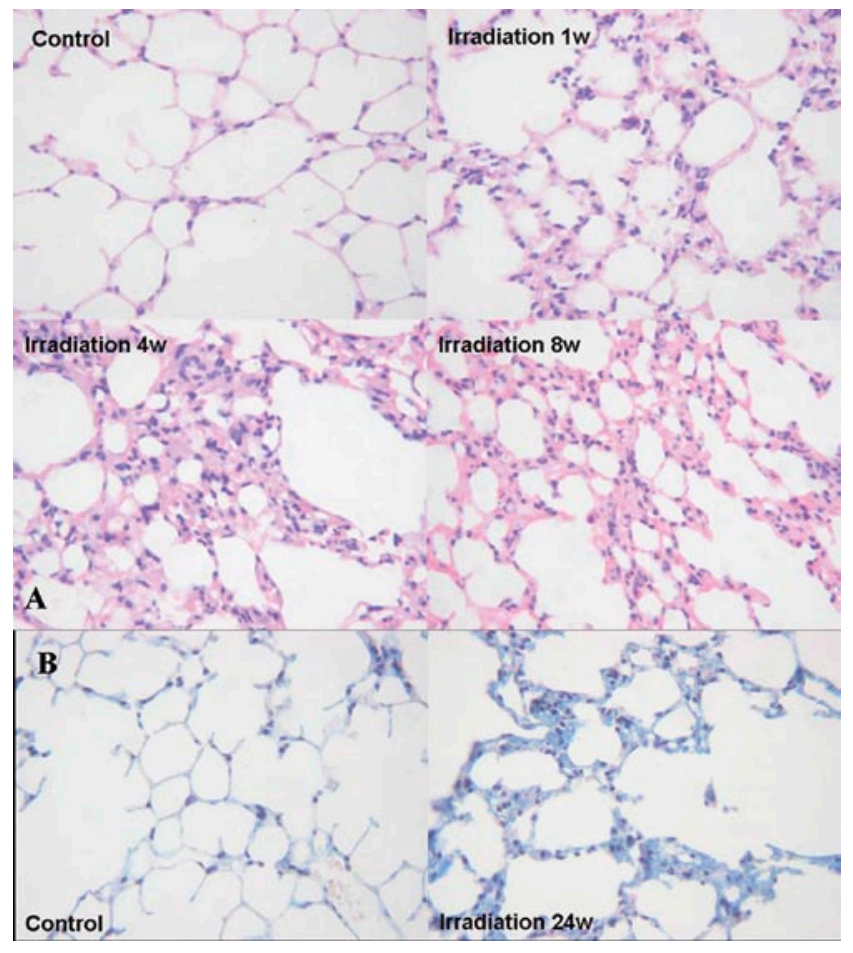

Figure 1. (A) H\&E staining for pathological features of radiation-induced lung injury in mouse lung tissues. Four representative slides indicate the control (control), 1 week p.i. (irradiation $1 w$ ), 4 weeks p.i. (irradiation 4 w), 8 weeks p.i. (irradiation 8 w). Original magnification, x200. (B) Masson's staining for collagen in mouse lung tissues of the two groups at 24 weeks Two representative slides are shown for the no treatment (control) and irradiated (irradiation 24w) groups. Collagen deposition (light blue stain). Original magnification, $\mathrm{x} 200$.

Western blot analysis. Cell extracts from the lung tissue samples were prepared using cold cell lysate radioimmunoprecipitation assay buffer (RIPA, Sigma-Aldrich, Shanghai, China). The protein concentration was determined using the micro-BCA kit (Applygen Technologies Inc., Beijing, China). According to the conventional methods modified by Fuentes et al (27), equal amounts of protein (50 $\mu \mathrm{g} /$ condition) from individual mice in each group were re-electrophoresed on $8-10 \%$ SDS-PAGE gel and then electrotransferred to polyvinylidene fluoride (PVDF, Millipore) membranes. The membranes were blocked by rocking with blocking buffer for $1 \mathrm{~h}$ at room temperature. The blots were then incubated overnight at $4^{\circ} \mathrm{C}$ with primary antibodies raised against GATA-3 (1:500) and Arg-1 (1:500), with $\beta$-actin (1:1000) used as an internal control. After 3 rinses with TBS-T buffer, the blots were incubated with horseradish peroxidase-conjugated anti-rabbit IgG Abs (1:3000 dilution in TBS-T) at room temperature for $1 \mathrm{~h}$ and washed with TBS-T buffer for $30 \mathrm{~min}$. Finally, the reaction was visualized with a chemiluminescence reagent kit (BioTime, Beijing, China) and exposed to Kodak Scientific Imaging Systems film. All primary antibodies were purchased from Santa Cruz Biotechnology.

Statistical analysis. Statistical analysis was performed with the SPSS software package (version 13.0) for Windows. All data are expressed as means \pm SD. Between group comparisons were performed using the Mann-Whitney U test, and the data for the various time points were analyzed with the independent samples t-test. A value of $\mathrm{P}<0.05$ was considered statistically significant.

\section{Results}

Histological changes. Following irradiation, histological changes of interstitial pneumonia were evident. At 1 week after radiation, lungs of the radiated mice showed mild structural changes. The initial injury included edema of the alveolar walls, intra-alveolar hemorrhage, alveolar exudation, neutrophil infiltration and interstitial edema. At 4 and 8 weeks p.i., continuied inflammation was evident. The alveolar septa were thickened, and the alveolar spaces were smaller (Fig. 1A). Large deposits of collagen were observed in the alveolar septa and bronchiolar area, with obliteration of the alveoli, particularly between 16 and 24 weeks (Fig. 1B).

\section{Immunohistochemistry}

Expression of Arg-1 in lung tissue. The staining patterns of Arg-1 in the lung tissues are shown in Fig. 2. Compared with 


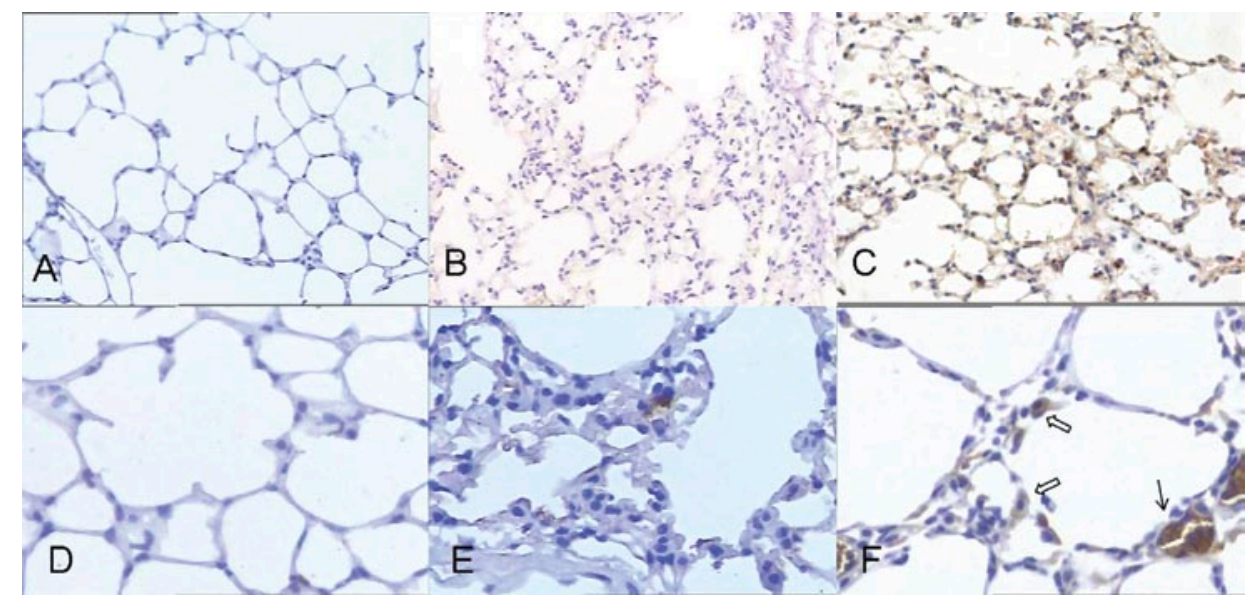

Figure 2. Differences in immunohistochemical stainings are shown for Arg-1 expression in mouse lung tissues for different treatments at different time points. Six representative slides are shown for the control and irradiated mice. (A and D) Control, (B and E) irradiated 2 weeks p.i., and (C and F) irradiated 16 weeks p.i. groups. (A-C, original magnification, x200; D-F, original magnification, x400). White arrows show alveolar macrophages and black arrow shows vascular endothelial cells in F.

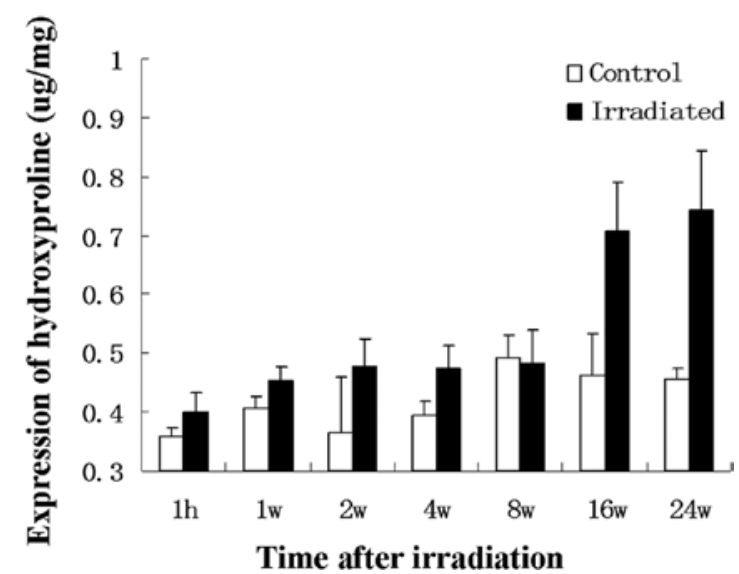

Figure 3. Time course and comparison of hydroxyproline expression in mouse lung tissues after irradiation.

the control, mild expression of Arg-1 was noted in the lung tissues at 2 weeks p.i. At 16 weeks p.i., the lung parenchyma had already revealed a pronounced increased expression of Arg-1, and vascular endothelial cells and alveolar macrophages may have served as important sources of Arg-1 expression. At the same time, there was evidence of interstitial fibrosis with accumulation of elastic fibers, deposition of collagen, and, consequently, destruction of normal tissue architecture.

Measurement of hydroxyproline content. In general measurements, the content of hydroxyproline in the radiation group was different from that in the control group $(\mathrm{P}<0.05)$. The irradiated mice expressed significantly increased amounts of hydroxyproline at 16 and 24 weeks $(0.708 \pm 0.081$ and $0.744 \pm 0.098 \mu \mathrm{g} / \mathrm{mg})(\mathrm{P}<0.01)$ (Fig. 3), compared to the mice in the control group.

ELISA analysis. Serum from the C57BL/6 mice treated with irradiation was analyzed for expression of IL-13 at different time points. In general, the irradiation group tended to express different levels of IL-13 compared to the control group

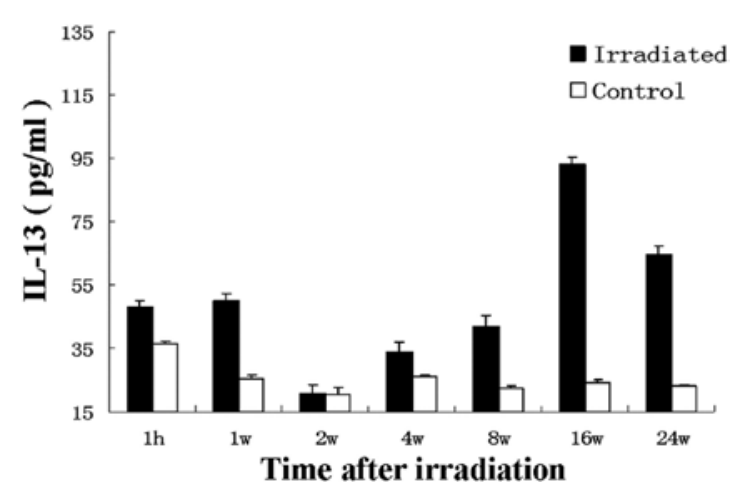

Figure 4. Time course and comparison of IL-13 expression in mouse serum after irradiation.

$(\mathrm{P}<0.01)$. Upon analysis for each time point, expression of IL-13 in the serum from the irradiated group was significantly greater than that in the control group at every time point, apart from 2 weeks p.i. $(\mathrm{P}<0.01)$. A maximal value $(92.958 \pm 2.488 \mathrm{pg} /$ $\mathrm{ml})$ appeared at 16 weeks p.i. $(\mathrm{P}<0.01)($ Fig. 4$)$.

\section{$R T$-PCR analyses}

GATA-3 mRNA expression. The GATA-3 mRNA expression in the different study groups are shown in Fig. 5A. There was a significantly statistical difference between the groups $(\mathrm{P}<0.01)$. The control group exhibited relative levels of GATA-3 mRNA expression between 0.017 and 0.03 . Radiation-induced GATA-3 was obviously increased and reached maximal values at 1 and 2 weeks p.i. (relative mRNA expression, $0.221 \pm 0.041$ and $0.179 \pm 0.032$, respectively) $(\mathrm{P}<0.01)$.

Arg-1 mRNA expression. There was no statistical difference between the groups $(\mathrm{P}>0.05)$. As shown in Fig. 5B, in contrast to the control group, Arg-1 mRNA was decreased at the early stage of radiation-induced lung injury, particularly at 2 and 4 weeks p.i. $(\mathrm{P}<0.05)$, but increased suddenly at 16 weeks p.i. (relative mRNA expression, $0.056 \pm 0.004$ ) and maintained a high level up to 24 weeks p.i. $(\mathrm{P}<0.01)$. 

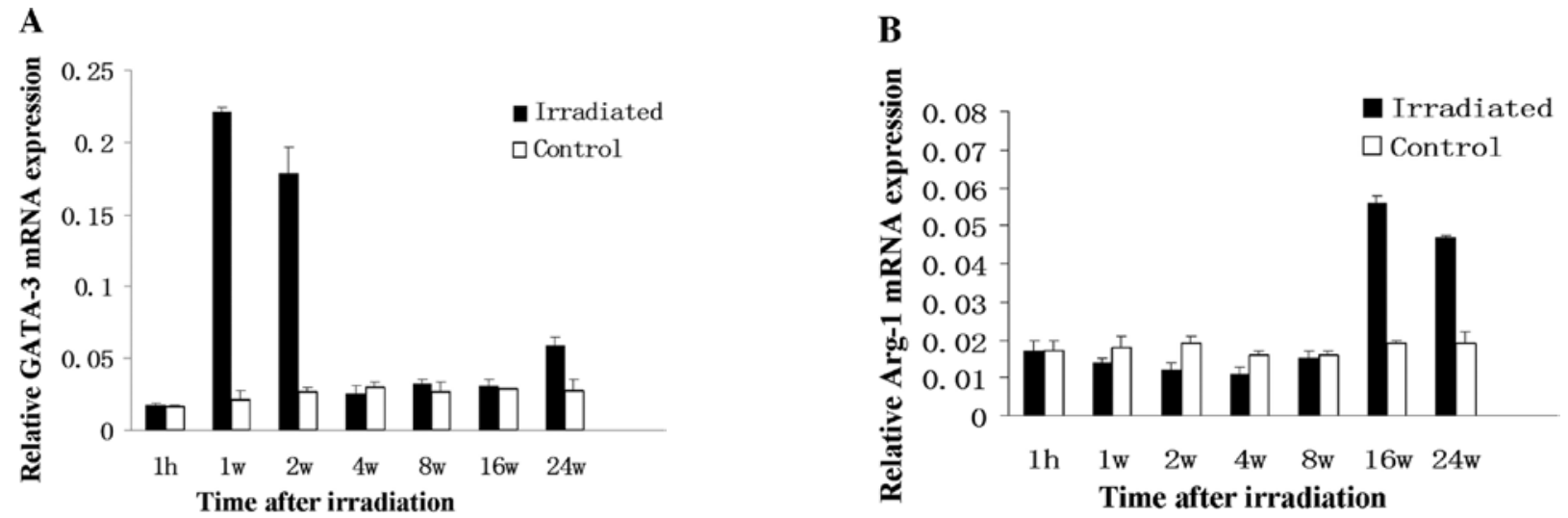

Figure 5. Time course and comparison of GATA-3 (A) and Arg-1 (B) relative mRNA expression in mouse lung tissues after irradiation. mRNA expression was determined from $1 \mathrm{~h}$ until 24 weeks (24w).

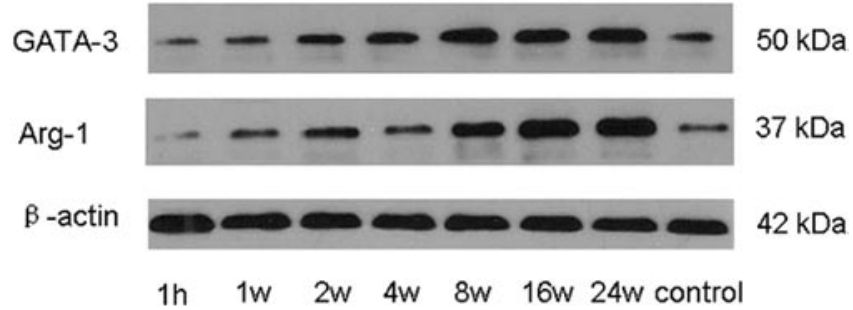

Figure 6. Western blot analysis was used to evaluate the protein expression of GATA-3 and Arg-1.

Western blot analysis. Fig. 6 shows the protein expression of GATA-3 and Arg-1. Compared with the control, GATA-3 protein expression was markedly increased at 2 weeks p.i. and was obviously increased from 4 weeks p.i.; particularly at 8 weeks p.i., Arg-1 appeared to produce more proteins than the control at 8 weeks p.i. and was obviously increased at 16 and 24 weeks p.i.

\section{Discussion}

Tissue fibrosis, which results in the destruction of normal organ function, is a leading cause of morbidity and mortality. The causes of fibrosis are diverse regardless of the tissue involve, and common features include the sequential recruitment of inflammatory cells, over-proliferation of matrix-producing cells, and the overproduction of extracellular matrix. Although the exact molecular mechanism leading to fibrosis is not yet clear, evidence from preclinical models strongly implicates Th2-specific signaling in the propagation of this process $(3,28)$. In this study, C57BL/6 mice received thoracic irradiation. Based on the results from changes in histology and the content of hydroxyproline the mouse model of RILF was successfully established. In general measurements, compared with the control group, the levels of Th2-like immune response-associated factors (GATA-3, IL-13 and Arg-1) and the hydroxyproline content in the irradiated mice were up-regulated. It appears that the Th2-like immune response is associated with RILF.

Arg-1 generates L-ornithine, an important precursor for proline that enhances collagen biosynthesis, promoting cell growth and tissue repair (21). In this study, we found that the peak level of hydroxyproline in irradiated lung tissues followed the peak level of Arg-1 expression. Arg-1 up-regulated expression of hydroxyproline in the lung tissues after irradiation to promote the progression of fibrotic tissue reactions. Arg-1 is a hallmark of alternative macrophage activation, which plays a role in fibrosis $(29,30)$. Upon immunohistochemistry, mild expression of Arg-1 was noted in the tissues at 2 weeks p.i. However, at 16 weeks p.i., the lung parenchyma had already revealed a pronounced increased expression of Arg-1, and Arg-1 was partly expressed in the macrophages. However, the vessel endothelial and other cells also exhibited positive staining of Arg-1. Similar observations of Arg-1 expression have been previously reported $(31,32)$. These results indicate that Arg-1 is the hallmark for alternatively activated macrophages and also for other mediators in other cells, and takes part in the pathogenesis of RILF. Thus, it can be used as an effective monitoring indicator for RILF.

Studies have reported $(30,33,34)$ that IL-13/IL-4 induced alternatively activated macrophages enhance the expression of Arg-1. In our study, we found that the increase in IL-13 expression in the serum occurred earlier than the up-regulated expression of Arg-1 mRNA and protein in the lung tissues. It has been proposed that Arg-1 may be regulated by Th-2 cytokines during RILF. GATA-3 has been found to regulate IL-13 in the development of the Th2 phenotype $(20,21)$. In our study, we found that GATA-3 was obviously increased and reached maximal values at 1 and 2 weeks p.i., and the peak level of IL-13 in the serum irradiated mice followed the peak level of GATA-3 expression, suggesting a causative relationship. GATA-3 may regulate the expression of Th2 cytokines during the phase of RILF. Moreover, the peak level of GATA-3 expression was noted earlier than any of the other factors (IL-13 and Arg-1) during RILF, suggesting that GATA-3 plays an important role in RILF and can be used as an effective predictor for RILF. From these observations, it seems that GATA-3 up-regulates expression of Arg-1 mRNA and protein by up-regulating the expression of Th2 cytokines during RILF.

In conclusion, the Th2-like immune response takes part in the promotion of pulmonary fibrosis, and GATA-3 plays a key role in this Th2-like immune response. Although a causal 
relationship between the Th2-like immune response and the pathogenesis of RILF cannot be definitively established from this study, it does indicate that restoration of the immunological balance probably represents an important therapeutic intervention strategy for the treatment of RILF. Thus, GATA-3 may be an important target for the treatment of RILF, which should be investigated in future studies.

\section{Acknowledgements}

This study was supported by grants from the National Natural Science Foundation of China (no. 30770653). The authors acknowledge Pui-Kai Li for an excellent critical review of the manuscript.

\section{References}

1. Marks LB, Bentzen SM, Deasy JO, Kong FM, Bradley JD, Vogelius IS, El Naqa I, Hubbs JL, Lebesque JV, Timmerman RD, Martel MK and Jackson A: Radiation dosevolume effects in the lung. Int J Radiat Oncol Biol Phys 76: 70-76, 2010.

2. Rübe CE, Uthe D, Wilfert F, Ludwig D, Yang K, König J, Palm J, Schuck A, Willich N, Remberger K and Rübe C: The bronchiolar epithelium as a prominent source of pro-inflammatory cytokines after lung irradiation. Int J Radiat Oncol Biol Phys 61: 1482-1492, 2005.

3. Brush J, Lipnick SL, Phillips T, Sitko J, McDonald JT and McBride WH: Molecular mechanisms of late normal tissue injury. Semin Radiat Oncol 17: 121-130, 2007.

4. Murphy KM and Reiner SL: The lineage decisions of helper T cells. Nat Rev Immunol 2: 933-944, 2002.

5. Abbas AK, Murphy KM and Sher A: Functional diversity of helper T lymphocytes. Nature 383: 787-793, 1996.

6. Wynn TA: Fibrotic disease and the TH1/TH2 paradigm. Nat Rev Immunol 4: 583-549, 2004

7. Lee CG, Homer RJ, Zhu Z, Lanone S, Wang X, Koteliansky V, Shipley JM, Gotwals P, Noble P, Chen Q, Senior RM and Elias JA: Interleukin-13 induces tissue fibrosis by selectively stimulating and activating transforming growth factor. J Exp Med 194: 809-821, 2001.

8. Xu J, Mora AL, LaVoy J, Brigham KL and Rojas M: Increased bleomycin-induced lung injury in mice deficient in the transcription factor T-bet. Am J Physiol Lung Cell Mol Physiol 291: 658-667, 2006.

9. Buttner C, Skupin A and Reimann T: Local production of interleukin-4 during radiation-induced pneumonitis and pulmonary fibrosis in rats: macrophages as a prominent source of interleukin-4. Am J Respir Cell Mol Biol 17: 315-325, 1997.

10. Huaux F, Liu T, McGarry B, Ullenbruch $M$ and Phan SH: Dual roles of IL-4 in lung injury and fibrosis. J Immunol 170: 2083-2092, 2003.

11. Tabata C, Kadokawa Y, Tabata R, Takahashi M, Okoshi K, Sakai Y, Mishima $\mathrm{M}$ and Kubo H: All-trans-retinoic acid prevents radiationor bleomycin-induced pulmonary fibrosis. Am J Respir Crit Care Med 174: 1352-1360, 2006.

12. Haase MG, Klawitter A, Geyer P and Baretton GB: Expression of the immunomodulator IL-10 in type I pneumocytes of the rat: alterations of IL-10 expression in radiation-induced lung damage. J Histochem Cytochem 55: 1167-1172, 2007.

13. Yang XB: Molecular and Cellular Mediators in Radiationinduced Lung Injury. The University of North Carolina at Chapel Hill, UMI Dissertation Publication 3354527, pp17, 2009.

14. Kehrer JP, Lee YC and Solem SM: Comparison of in vitro and in vivo rates of collagen synthesis in normal and damaged lung tissue. Exp Lung Res 10: 197-201, 1986.

15. Ko LJ and Engel JD: DNA-binding specificities of the GATA transcription factor family. Mol Cell Biol 13: 4011-4022, 1991.
16. Chakir H, Wang H, Lefebvre DE, Webb J and Scott FW: T-bet/ GATA-3 ratio as a measure of the Th1/Th2 cytokine profile in mixed cell populations: predominant role of GATA-3. J Immunol Methods 278: 157-169, 2003.

17. Park SW, Ahn MH, Jang HK, Jang AS, Kim DJ, Koh ES, Park JS, Uh ST, Kim YH, Park JS, Paik SH, Shin HK, Youm W and Park CS: Interleukin-13 and its receptors in idiopathic interstitial pneumonia: clinical implications for lung function. J Korean Med Sci 24: 614-620, 2009.

18. Reiman RM, Thompson RW, Feng CG, Hari D, Knight R, Cheever AW, Rosenberg HF and Wynn TA: Interleukin-5 (IL-5) augments the progression of liver fibrosis by regulating IL-13 activity. Infect Immun 74: 1471-1479, 2006.

19. Kishikawa H, Sun J, Choi A, Miaw SC and Ho IC: The cell type-specific expression of the murine IL-13 gene is regulated by GATA-3. J Immunol 167: 4414-4420, 2001.

20. Lavenu-Bombled C, Trainor CD, Makeh I, Romeo PH and Max-Audit I: Interleukin-13 gene expression is regulated by GATA-3 in T cells: role of a critical association of a GATA and two GATG motifs. J Biol Chem 277: 18313-18321, 2002.

21. Hesse M, Modolell M, La Flamme AC, Schito M, Fuentes JM, Cheever AW, Pearce EJ and Wynn TA: Differential regulation of nitric oxide synthase- 2 and arginase-1 by type 1/type 2 cytokines in vivo: granulomatous pathology is shaped by the pattern of L-arginine metabolism. J Immunol 167: 6533-6544, 2001.

22. Kitowska K, Zakrzewicz D, Königshoff M, Chrobak I, Grimminger F, Seeger W, Bulau P and Eickelberg O: Functional role and species-specific contribution of arginases in pulmonary fibrosis. Am J Physiol Lung Cell Mol Physiol 294: 34-45, 2008.

23. Wei LH, Jacobs AT, Morris SM Jr and Ignarro LJ: IL-4 and IL-13 upregulate arginase I expression by cAMP and JAK/ STAT6 pathways in vascular smooth muscle cells. Am J Physiol Cell Physiol 279: 248-256, 2000.

24. Kropf P, Fuentes JM, Fähnrich E, Arpa L, Herath S, Weber V, Soler G, Celada A, Modolell M and Müller I: Arginase and polyamine synthesis are key factors in the regulation of experimental leishmaniasis in vivo. FASEB J 19: 1000-1002, 2005.

25. Lange $M$ and Malyusz M: Improved determination of small amounts of free hydroxyproline in biological fluids. Clin Chem 40: 1735-1738, 1994

26. Liu W and David AS: Validation of a quantitative method for real time kinetics. Biochem Biophys Res Commun 294: 347-353, 2002.

27. Fuentes JM, Lompré AM, Møller JV, Falson P and le Maire M: Clean Western blots of membrane proteins after yeast heterologous expression following a shortened version of the method of Perini et al. Anal Biochem 285: 276-278, 2000.

28. Sime PJ and O'Reilly KM: Fibrosis of the lung and other tissues: new concepts in pathogenesis and treatment. Clin Immunol 99: 308-319, 2001.

29. David MM: The many faces of macrophage activation. J Leukoc Biol 73: 209-212, 2003.

30. Mosser DM and Edwards JP: Exploring the full spectrum of macrophage activation. Nat Rev Immunol 8: 958-969, 2008.

31. Mora AL, Torres-González E, Rojas M, Corredor C, Ritzenthaler J, Xu J, Roman J, Brigham K and Stecenko A: Activation of alveolar macrophages via the alternative pathway in herpesvirus-induced lung fibrosis. Am J Respir Cell Mol Biol 35: 466-473, 2006.

32. Munder M, Eichmann K, Morán JM, Centeno F, Soler G and Modolell M: Th1/Th2-regulated expression of arginase isoforms in murine macrophages and dendritic cells. J Immunol 163: 3771-3777, 1999.

33. Mantovani A, Sica A, Sozzani S, Allavena P, Vecchi A and Locati M: The chemokine system in diverse forms of macrophage activation and polarization. Trends Immunol 125: 677-686, 2004.

34. Boscá L, Zeini M, Través PG and Hortelano S: Nitric oxide and cell viability in inflammatory cells: a role for NO in macrophage function and fate. Toxicology 208: 249-258, 2005. 